\title{
Candidate Susceptibility Genes for Powdery and Downy Mildew in Watermelon and Squash
}

\author{
Ryan Porterfield and Geoffrey Meru* \\ Horticultural Sciences Department and Tropical Research and Education Center, University of Florida, IFAS 18905 SW $280^{\text {th }}$ St, Homestead, FL 33031, USA
}

"Corresponding author: Geoffrey Meru, Horticultural Sciences Department and Tropical Research and Education Center, University of Florida, IFAS 18905 SW 280 th St, Homestead, FL 33031, USA, Tel: +17862179287, E-mail: gmeru@ufl.edu

Receiving date: Sep 17, 2017; Acceptance date: Sep 28, 2017; Publication date: Oct 05, 2017

Copyright: ( 2017 Porterfield R, et al. This is an open-access article distributed under the terms of the Creative Commons Attribution License, which permits unrestricted use, distribution, and reproduction in any medium, provided the original author and source are credited.

\begin{abstract}
Powdery mildew (PM) caused by Podosphaera xanthii and downy mildew (DM) caused by Pseudoperonospora cubensis are two of the most economically important diseases for watermelon (Citrullus lanatus) and squash (Cucurbita pepo, C. maxima and C. moschata). Traditional breeding for resistance to PM and DM is resource intensive, often requiring decades' long phenotyping and selection processes. As an alternative, durable and broadspectrum resistance to PM and DM can be obtained through loss-of-function of susceptibility genes in elite breeding material. Susceptibility genes for PM [Mildew-Locus-O (MLO) and Powdery Mildew Resistance (PMR)] and DM [Downy Mildew Resistance (DMR)] have been functionally proven in model plant species. Previous studies have reported candidate MLO genes for $C$. lanatus and C. pepo, but none for C. maxima and C. moschata. On the contrary, no PMR or DMR candidate genes have been identified for $C$. lanatus or any of the Cucurbita species. The current study used bioinformatics approaches based on sequence similarity, phylogenetic relationships and presence of conserved domains to predict candidate MLO genes in C. maxima and C. moschata and PMR and DMR genes in C. lanatus, C. pepo, C. maxima and C. moschata. Four MLO homologs in C. maxima and five in C. moschata clustered within Clade V, a clade containing all MLO susceptibility genes in dicots, and had highly conserved transmembrane domains and C-terminal PM interaction motif. Sixty-three candidate PMR genes were identified among the four species, 16 of which had close similarity to functionally proven PMR homologs in model species. Similarly, 37 candidate DMR genes were identified 12 among which clustered with functionally proven DMR homologs in model species. Functional analysis of the genes identified in the current study will reveal their role in pathogenesis and assess their potential for manipulation through gene editing methods to generate novel resistant plant genotypes.
\end{abstract}

Keywords: Powdery mildew; Downy mildew; MLO; DMR; PMR; Watermelon; Squash

\section{Introduction}

Watermelon (Citrullus lanatus) and squash (Cucurbita pepo, C. maxima and $C$. moschata) are economically important cucurbits in the U.S. with a combined annual value of approximately 0.75 billion dollars [1]. Powdery mildew (PM) caused by Podosphaera xanthii and downy mildew (DM) caused by Pseudoperonospora cubensis are economically important diseases for the two vegetable crops [2] and can cause significant yield and quality losses to growers if not properly managed. Strict preventative fungicide spray regimens are often required to keep plants healthy and free from disease [3]. Although effective, persistent use of fungicides poses toxicity hazards to humans and non-target organisms, as well as a risk for fungicide-resistance [4-6]. Genetic resistance is the most preferred management option for the two diseases. However, traditional breeding for PM and DM resistance is resource intensive, often requiring decades' long phenotyping and selection processes.

Knockout of susceptibility genes, also known as S-genes, may be a rapid and effective method for conferring resistance in cultivated plant species. Inactivation of S-genes through gene-knockout, geneknockdown or virus induced gene-silencing has been proven a viable option for generating resistance genotypes against important diseases such as rice blast in rice (Oryza sativa) [7], citrus canker in citrus
(Citrus paradisi) [8] and powdery mildew in grapevine (Vitis vinifera) [9], tomato and pepper [10]. Currently, two classes of PM S-genes are known, Mildew-Locus-O (MLO) genes and Powdery Mildew Resistance (PMR), both of which are loss-of-function genes [11-12]. Since the first discovery of MLO genes in barley in 1942, many MLOlike genes have been described and annotated in several model species including Arabidopsis thaliana and Solanum lycopersicum [11-12]. Phylogenetic analysis of MLO proteins reveal as many as 6 distinct clades, denoted clades I through VI, and Clade V harbors all the proven MLO susceptibility genes for PM in dicots [11]. MLO genes are characterized by 7 transmembrane helices, similar to metazoan and fungal G-protein coupled receptors [13]. All the MLO susceptibility genes in clade $\mathrm{V}$ share highly conserved domains within transmembrane portions of the protein and harbor a four amino acid long motif at the C-terminus that has been associated with PM interaction [14]. Functional clade V MLO proteins negatively regulate defense pathways at the site of PM inoculation, thus allowing the pathogen to induce pathogenesis [11]. Therefore, a loss of function in MLO genes consequently induce resistance to PM [10,15]. PMR genes on the other hand are far less studied and most are putative, but some have been shown to play a role in PM susceptibility in Arabidopsis (AtPMR4, AtPMR5, and AtPMR6) and tomato (SlPMR4) [16-19]. PMR genes are involved in cell wall biology where they mediate structure formation and pectin accumulation [18]. In Arabidopsis, loss-of-function in AtPMR4, which encodes callose synthase, resulted in PM resistant genotypes [16]. 
Page 2 of 14

Two S-genes for downy mildew, DMR1 and DMR6, have been described in Arabidopsis and tomato [12,19-21]. dmr1 and dmr6 mutants induce resistance to DM by accumulating elevated levels of homoserine [21] and 2OG-Fe(II) oxygenase [20], respectively.

In cucurbits, MLO candidate genes have been identified in cucumber (Cucumis sativus [12], watermelon (C. lanatus), melon (Cucumis melo) and squash (C. pepo) [22]. However, no MLO candidate genes have been identified for C. maxima or C. moschata. Similarly, information on candidate genes for PMR and DMR in $C$. lanatus, $C$. pepo, C. maxima and C. moschata is currently lacking. Identification of MLO, PMR and DMR genes across the Cucurbitaceae family is an indispensable prerequisite for fundamental studies into the functional role of candidate genes in pathogenesis, and subsequently, identification of potential targets for genetic manipulation to generate novel resistant plant genotypes. Therefore, the objective of the current study was to use bioinformatics approaches based on sequence similarity, phylogenetic relationships and presence of conserved domains to predict candidate MLO genes in $C$. maxima and $C$. moschata and PMR and DMR genes in C. lanatus, C. pepo, C. maxima and C. moschata.

\section{Materials and Methods}

\section{Identification of MLO, PMR and DMR homologs and their chromosomal locations}

Amino acids sequences for MLO genes in dicots Arabidopsis (Arabidopsis thaliana, AtMLO1-AtMLO15), Capsicum annuum (CaMLO2), Solanum lycopersicum (SlMLO1), Pisum sativus (PsMLO1), Medicago truncatula (MtMLO1), Lotus japonicas (LjMLO1), Vitis vinifera (VvMLO1) and Cucumis sativus (CsaMLO1, CsaMLO8 and CsaMLO11)], and monocots [Hordeum vulgare (HvMLO), Triticum aestivum (TaMLO_A1b and TaMLO_B1) and Oryza sativa (OsMLO2 and OsMLO3)], PMR genes in Arabidopsis (AtPMR4, AtPMR5 and AtPMR6), tomato (SlPMR4) and cucumber (CsaPMR4-2 and CsaPMR4-9), as well as DMR genes in Arabidopsis (AtDMR1 and AtDMR6), tomato (SlDMR1) and cucumber (CsaDMR6-1 and CsaDMR6-2), were collected from the NCBI protein database and the cucurbit genomics database.

Each dicot MLO protein was used in a blast search against the $C$. maxima and $C$. moschata genomes, while PMR and DMR proteins were searched against $C$. lanatus, $C$. pepo, $C$. maxima and $C$. moschata genomes (http://cucurbitgenomics.org/). For C. lanatus, C. maxima and $C$. moschata, a blastp search was used and the top five results were saved after removal of duplicates. However, since blastp function is not available for $C$. pepo, a tblastn search was performed against the unigene database (v1.0). The chromosomal distribution of MLO, PMR and DMR protein homologs across the C. lanatus, C. pepo, C. maxima and $C$. moschata genomes was visualized using Mapchart [23].

\section{Sequence alignment, phylogenetic analysis and clade annotation}

All MLO (monocots and dicots), PMR and DMR protein sequences from model species were aligned with protein sequences extracted from C. lanatus, C. pepo, C. maxima and C. moschata genomes using the ClustalW alignment program in MEGA6 software [24]. The neighbor-joining clustering method was used to generate bootstrap consensus phylogenetic trees using 100 replicates. For MLO genes, clades were annotated as described by Devoto et al. [25].

\section{Conserved domain analysis of MLO proteins}

All MLO-like sequences from C. maxima and C. moschata that clustered in clade $\mathrm{V}$ were aligned to functionally proven MLO proteins in dicots to confirm presence of conserved domains in the transmembrane protein (TM1 - TM7), and the PM interaction Cterminus (D/E-F-S/T-F) using ClustalO alignment with boxshade $[14,26]$. To build a MLO consensus sequence, an amino acid was regarded as conserved if at least 7 out of 8 of the functionally proven MLO proteins harbored it, or an amino acid with similar chemical properties as determined by the Rasmol color scheme [12,27]. The number of amino acids deviating from the conserved sequence in each of the candidate proteins as well as in all the clade $\mathrm{V}$ proteins was counted to determine degree of similarity.

\section{Results and Discussion}

\section{MLO homologs, clade annotation and conserved domains}

The search for MLO homologs revealed five unique proteins each in C. maxima (CmaMlO1, CmaMlO2, CmaMlO3, CmaMlO4 and CmaMLO5) and $C$. moschata (CmaMLO1, CmoMLO2, CmoMLO3, CmoMLO4 and CmoMLO5) (Table 1).

\begin{tabular}{|l|l|}
\hline Homolog & Designation in genome database \\
\hline Cucurbita maxima & CmaCh18G008880.1 \\
\hline CmaMLO1 & CmaCh02G008830.1 \\
\hline CmaMLO3 & CmaCh04G013590.1 \\
\hline CmaMLO4 & CmaCh13G001880.1 \\
\hline CmaMLO5 & CmaCh20G004770.1 \\
\hline Cucurbita moschata & \\
\hline CmoMLO1 & CmoCh18G008900.1 \\
\hline CmoMLO2 & CmoCh02G008830.1 \\
\hline CmoMLO3 & CmoCh04G014330.1 \\
\hline CmoMLO4 & CmoCh20G005110.1 \\
\hline CmoMLO5 & CmoCh13G001880.1 \\
\hline
\end{tabular}

Table 1: Mildew-locus-O homologs and their designation in the Cucurbita maxima and Cucurbita moschata genome databases.

All the ten homologs clustered in Clade V, a clade containing all MLO susceptibility genes in dicots (Figure 1). A similar study by Lovieno et al. [22] identified 14 MLO homologs in C. lanatus and 18 in C. pepo, three of which clustered in Clade $\mathrm{V}$ in the former and latter, respectively. In cucumber, Schouten et al. [12] identified 14 MLO-like proteins, 3 of which placed within clade V. Examination of the transmembrane portions of the MLO proteins as well as the PM interaction motif at the C-terminus (D/E-F-S/T-F) revealed a total of 119 conserved amino acids in the consensus sequence. Alignment of the consensus sequence to the functionally proven MLO proteins in clade $\mathrm{V}$ revealed high similarity, ranging from $94.8 \%$ to $100 \%$ (Table 2 and Figure 2). High level of similarity among clade $\mathrm{V}$ proteins has been noted in other plant species including cucumber [12], apricots (Prunus 
Citation: Porterfield R, Meru G (2017) Candidate Susceptibility Genes for Powdery and Downy Mildew in Watermelon and Squash. J Phylogenetics Evol Biol 5: 186. doi:10.4172/2329-9002.1000186

Page 3 of 14

armeniaca), peaches (Prunus persica), apples (Malus domestica) and strawberry (Fragaria xananassa) [11]. Although CmaMLO1 homolog in $C$. maxima clustered in Clade $\mathrm{V}$, it is likely not a PM susceptibility gene since it lacks the C-terminus PM interaction motif, and is annotated as an ubiquitin-conjugating enzyme on the genome database (http://cucurbitgenomics.org/).

\begin{tabular}{|l|l|l|}
\hline Homolog & $\begin{array}{l}\text { Number of deviating amino } \\
\text { acids }\end{array}$ & $\begin{array}{l}\text { Similarity } \\
\text { consensus sequence } \\
(\%)\end{array}$ \\
\hline AtMLO2 & 2 & 98.3 \\
\hline AtMLO6 & 3 & 97.5 \\
\hline AtMLO12 & 2 & 98.3 \\
\hline PsMLO1 & 2 & 98.3 \\
\hline MtMLO1 & 2 & 98.3 \\
\hline LjMLO1 & 6 & 94.9 \\
\hline SIMLO1 & 0 & 100 \\
\hline CaMLO2 & 0 & 100 \\
\hline CmaMLO2 & 2 & 98.7 \\
\hline CmaMLO3 & 7 & 95.5 \\
\hline CmaMLO4 & 1 & 99.4 \\
\hline CmaMLO5 & 1 & 99.4 \\
\hline CmoMLO1 & 3 & 98.1 \\
\hline CmoMLO2 & 2 & 98.7 \\
\hline CmoMLO3 & 8 & 94.8 \\
\hline CmoMLO4 & 1 & 99.4 \\
\hline CmoMLO5 & 1 & 99.4 \\
\hline
\end{tabular}

Table 2: Similarity in amino acid sequence within the transmembrane proteins and the C-terminus motif portions of clade V MLO proteins.

\section{PMR and DMR phylogenetic analysis}

Sixteen PMR-like homologs were identified in watermelon, five each for PMR4 and PMR5, and six for PMR6 (Table 3). Of these, one homolog each for PMR4 (ClaPMR4-1) and PMR5 (ClaPMR5-1), and two homologs for PMR6 (ClaPMR6-2 and ClaPMR6-3) clustered with PMR genes in Arabidopsis, cucumber and tomato (Figure 3A-C). For C. pepo fifteen PMR-like homologs were identified, five each for PMR4, PMR5 and PMR6 (Table 3). However, only CpePMR4-4 and CpePMR5-3 were closest in similarity to PMR genes in model species (Figure 3A- C). Thirty-two PMR-like homologs for $C$. maxima and $C$. moschata were identified, ten among which clustered with PMR genes in Arabidopsis, cucumber and tomato (Table 3). These candidate genes included four PMR4 (CmaPMR4-1, CmaPMR4-2, CmoPMR4-1 and CmoPMR4-2), two PMR5 (CmaPMR5-1 and CmoPMR5-1) and four PMR6 (CmaPMR6-4, CmaPMR6-5, CmoPMR6- 2 and CmoPMR6-4) homologs (Figure 3A-C). In cucumber, Schouten et al. [12] found 10 PMR-like homologs for PMR4, 1 for PMR5 and 13 for PMR6, none of which was differentially expressed following challenge with PM pathogen.

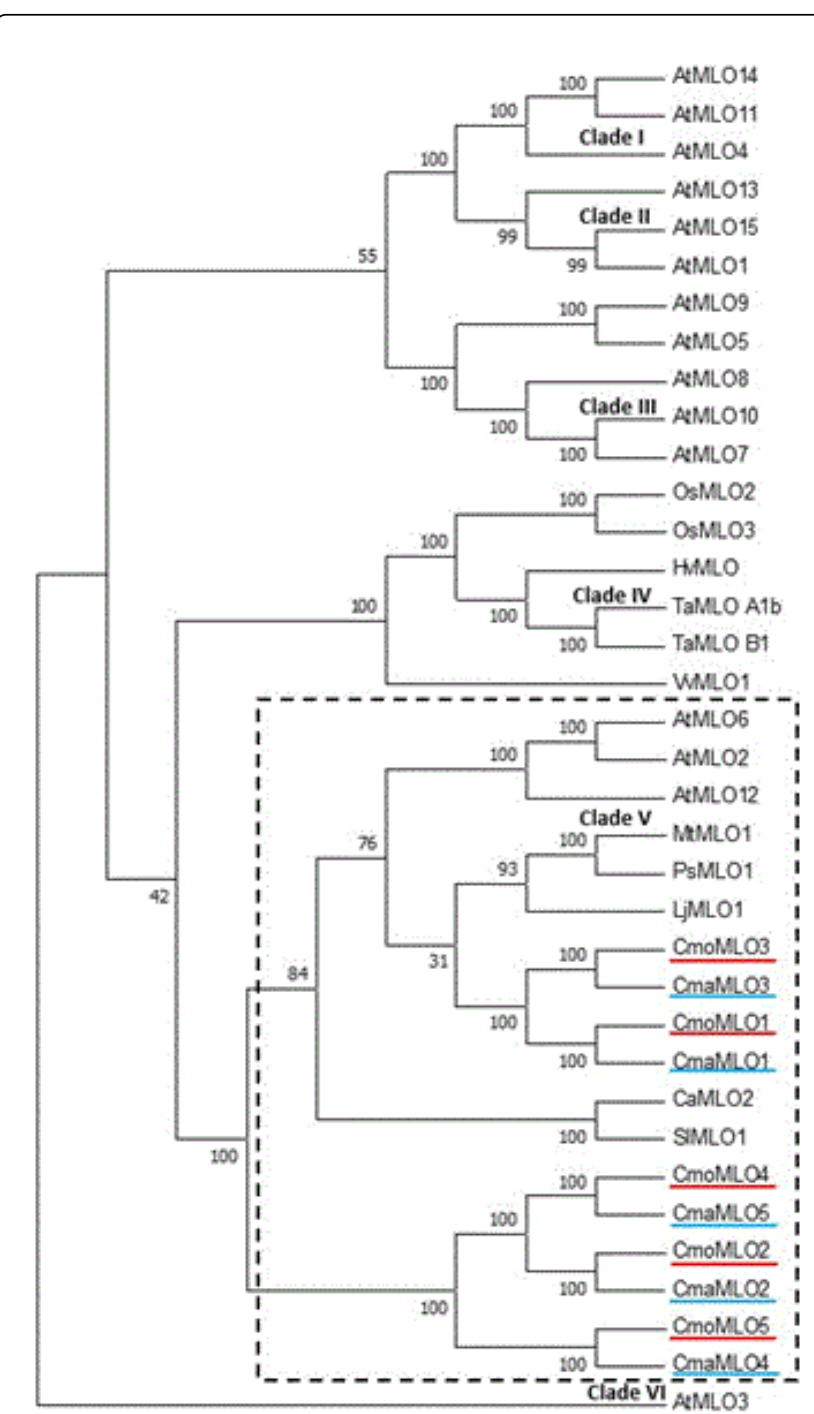

Figure 1: Mildew-locus-O phylogenetic analysis. Phylogenetic relationships and clade annotation of MLO-like proteins in Cucurbita moschata and Cucurbita maxima and other functionally proven MLO proteins. Clade IV and V represent MLO proteins in monocots and dicots (within dotted box), respectively. Proteins underlined in red (C. moschata) and blue ( $C$. maxima) represent those closest in similarity to functionally proven MLO proteins in dicots.

Thirty-seven DMR homologs were identified across the four species, seven for DMR-1 and thirty for DMR-6 (Table 4). For DMR-1, one candidate gene each for $C$. lanatus (ClaDMR1), $C$. pepo (CpeDMR1-2), C. maxima (CmaDMR1-2), and C. moschata (CmoDMR1-2) clustered with DMR-1 proteins in Arabidopsis, cucumber and tomato (Figure 4A-B). Similarly, two candidate DMR-6 genes each for $C$. lanatus (ClaDMR6-1 and ClaDMR6-2), C. maxima (CmaDMR6-1 and CmaDMR6-2), and C. moschata (CmoDMR6-1and CmoDMR6-2), and one for C. pepo (CpeDMR6-9) clustered with DMR genes in Arabidopsis and cucumber (Figure 4A-B). These results 
Citation: Porterfield R, Meru G (2017) Candidate Susceptibility Genes for Powdery and Downy Mildew in Watermelon and Squash. J Phylogenetics Evol Biol 5: 186. doi:10.4172/2329-9002.1000186

Page 4 of 14

are similar to those found in cucumber where one DMR1 genes and two DMR6 genes were found [12].

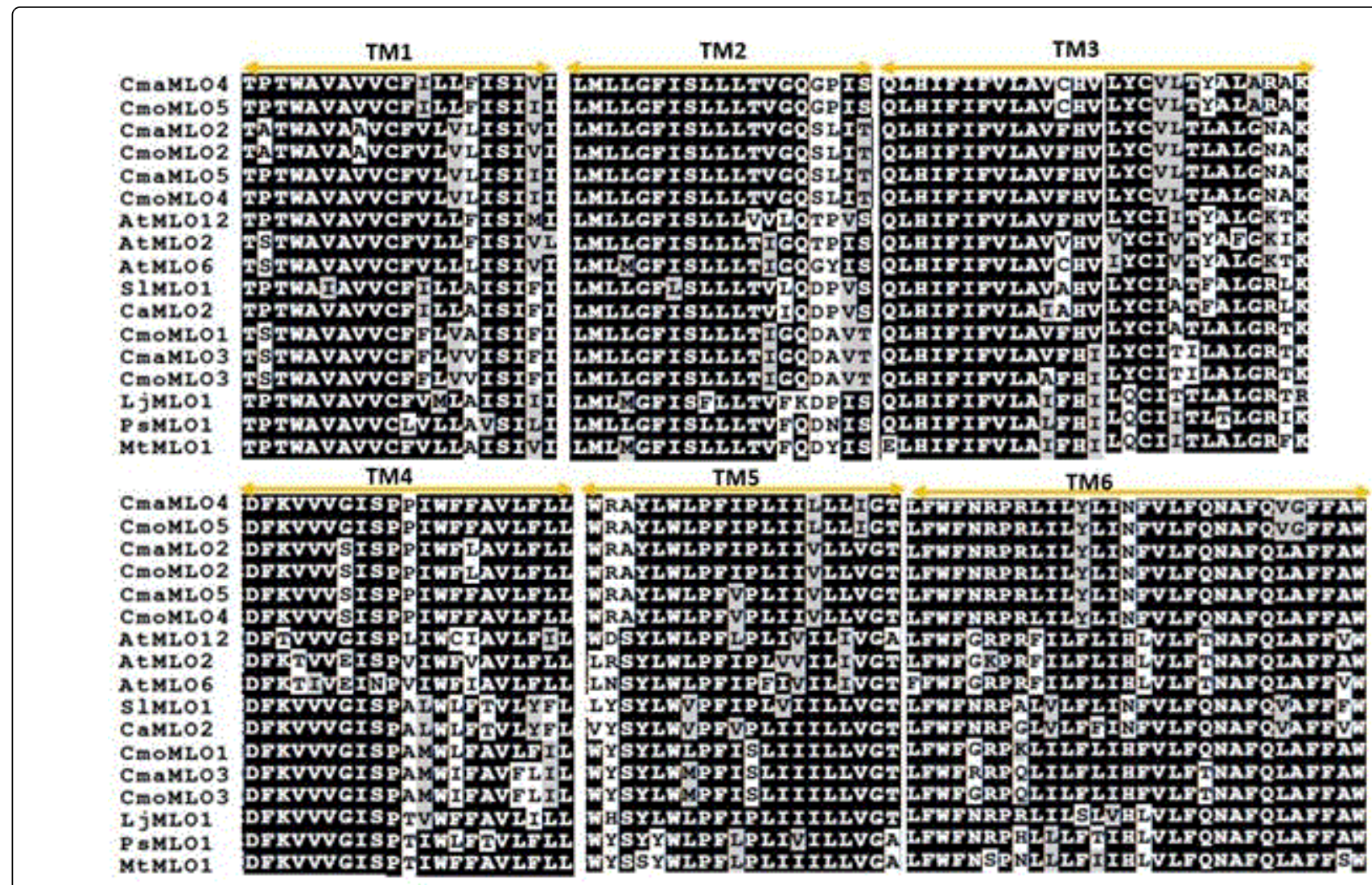

TM7

C-terminus motif

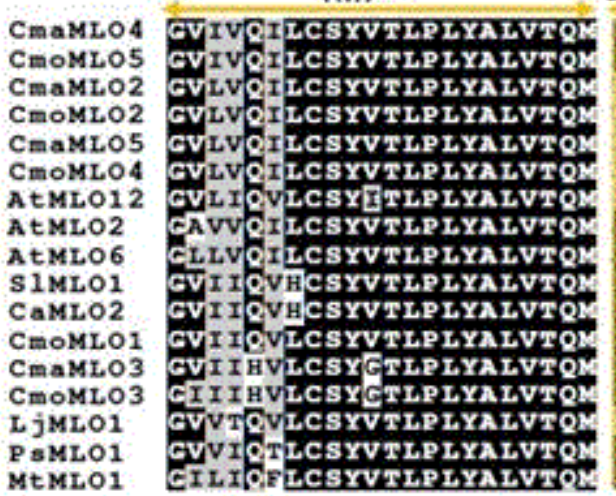

Figure 2: Alignment of conserved sequences. ClustalO alignment with boxshade of transmembrane proteins and C-terminus motif in Cucurbita moschata and Cucurbita maxima homologs and functionally proven MLO homologs in clade V. Orange shading indicates the Cterminus motif involved in powdery mildew interaction. 
Citation: Porterfield R, Meru G (2017) Candidate Susceptibility Genes for Powdery and Downy Mildew in Watermelon and Squash. J Phylogenetics Evol Biol 5: 186. doi:10.4172/2329-9002.1000186

Page 5 of 14

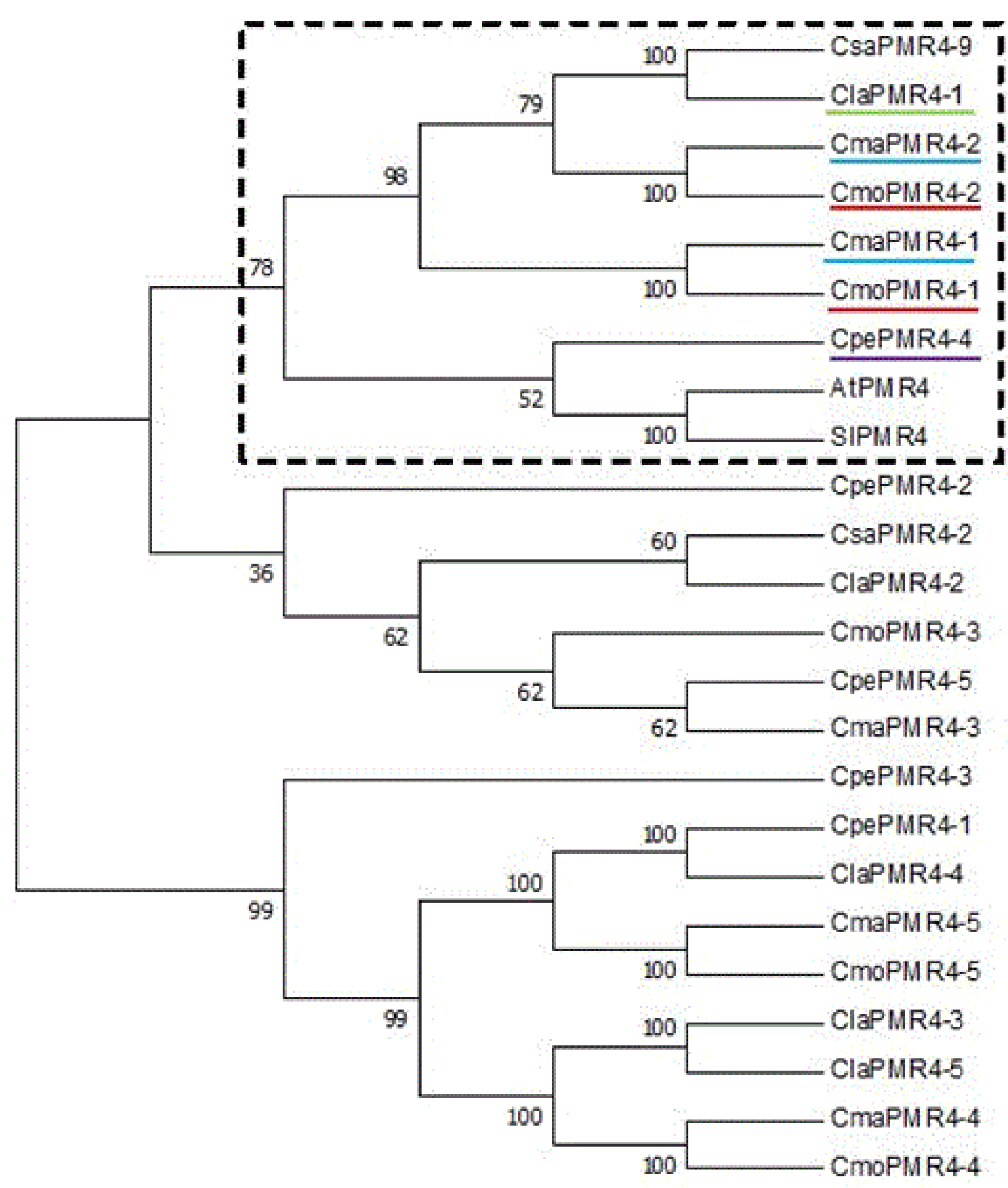

Figure 3A: Powdery Mildew Resistance phylogenetic analysis. Phylogenetic tree for PMR4 in Arabidopsis (AtPMR4, AtPMR5 and AtPMR6), tomato (SlPMR4) and cucumber (CsaPMR4-2, CsaPMR4-9, CsaPMR5, CsaPMR6-5, CsaPMR6-12 and CsaPMR6-13) and PMR-like proteins in Citrullus lanatus, Cucurbita pepo, Cucurbita maxima and Cucurbita moschata. Underlined proteins represent those closest in similarity to functionally proven PMR genes (within dotted box). 
Citation: Porterfield R, Meru G (2017) Candidate Susceptibility Genes for Powdery and Downy Mildew in Watermelon and Squash. J Phylogenetics Evol Biol 5: 186. doi:10.4172/2329-9002.1000186

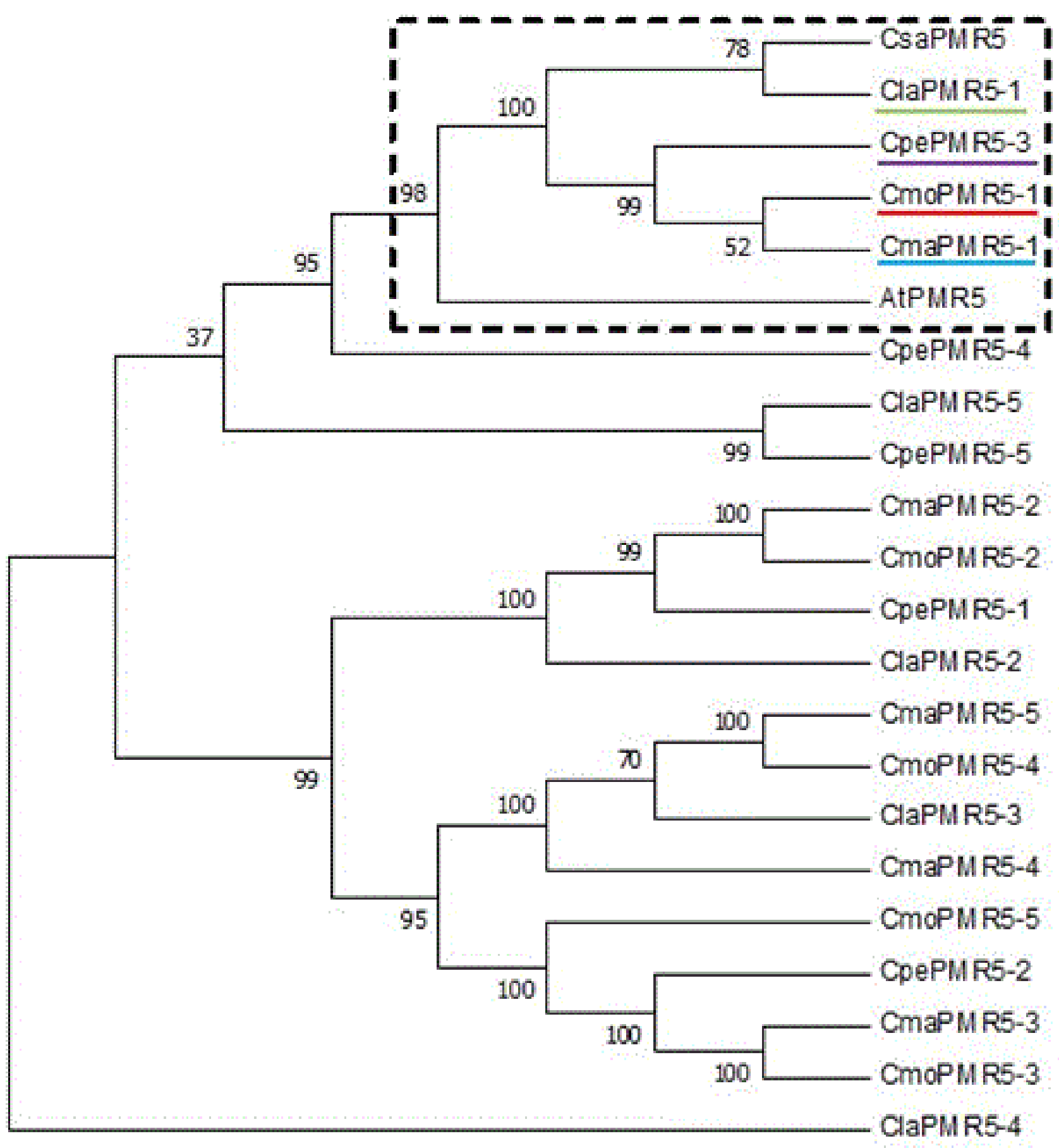

Figure 3B: Powdery Mildew Resistance phylogenetic analysis. Phylogenetic tree for PMR5 in Arabidopsis (AtPMR4, AtPMR5 and AtPMR6), tomato (SIPMR4) and cucumber (CsaPMR4-2, CsaPMR4-9, CsaPMR5, CsaPMR6-5, CsaPMR6-12 and CsaPMR6-13) and PMR-like proteins in Citrullus lanatus, Cucurbita pepo, Cucurbita maxima and Cucurbita moschata. Underlined proteins represent those closest in similarity to functionally proven PMR genes (within dotted box). 
Citation: Porterfield R, Meru G (2017) Candidate Susceptibility Genes for Powdery and Downy Mildew in Watermelon and Squash. J Phylogenetics Evol Biol 5: 186. doi:10.4172/2329-9002.1000186

Page 7 of 14

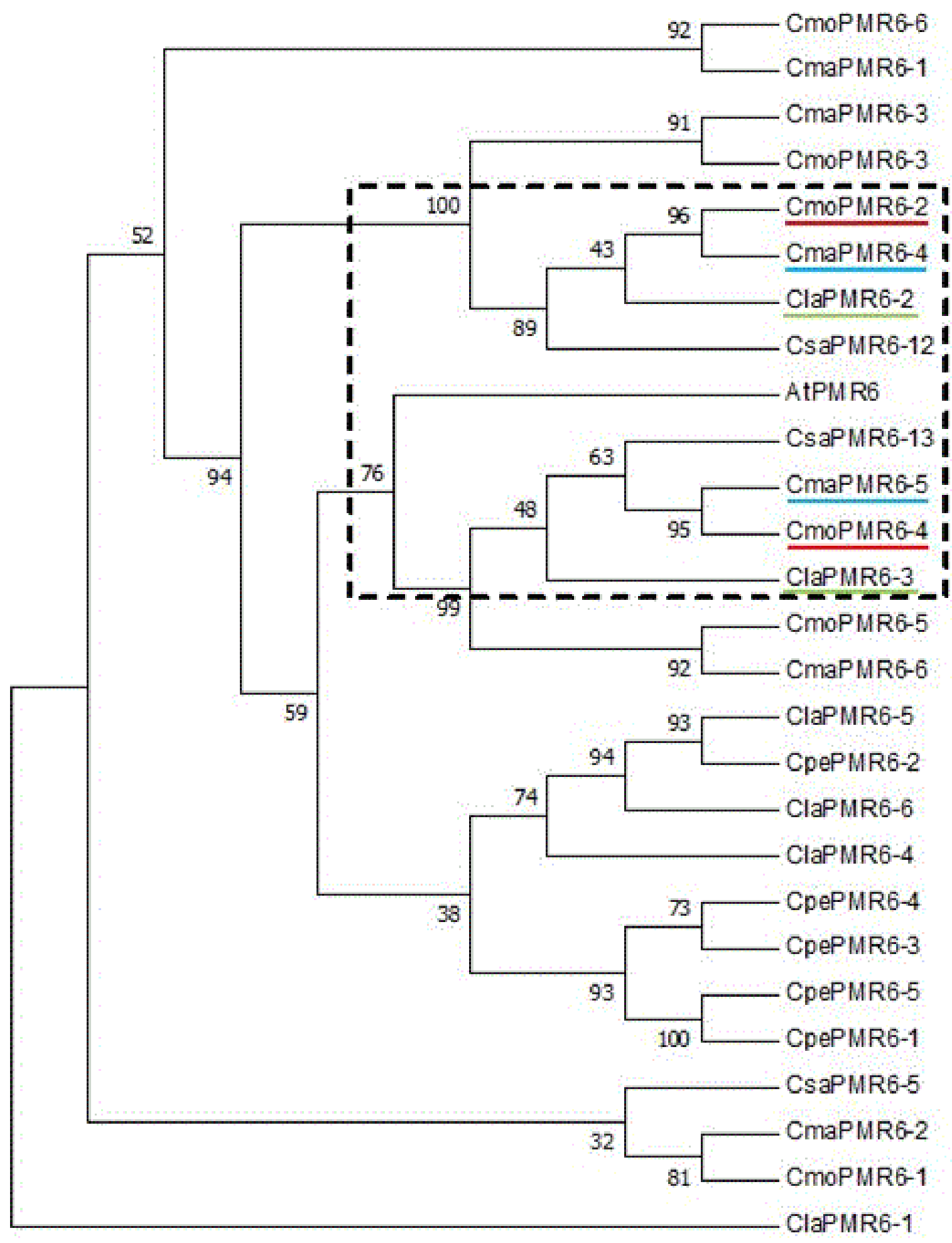

Figure 3C: Powdery Mildew Resistance phylogenetic analysis. Phylogenetic tree for PMR6 proteins in Arabidopsis (AtPMR4, AtPMR5 and AtPMR6), tomato (SlPMR4) and cucumber (CsaPMR4-2, CsaPMR4-9, CsaPMR5, CsaPMR6-5, CsaPMR6-12 and CsaPMR6-13) and PMRlike proteins in Citrullus lanatus, Cucurbita pepo, Cucurbita maxima and Cucurbita moschata. Underlined proteins represent those closest in similarity to functionally proven PMR genes (within dotted box). 
Citation: Porterfield R, Meru G (2017) Candidate Susceptibility Genes for Powdery and Downy Mildew in Watermelon and Squash. J Phylogenetics Evol Biol 5: 186. doi:10.4172/2329-9002.1000186

Page 8 of 14

\begin{tabular}{|c|c|c|c|c|}
\hline & Homolog & Gene & Homolog & Gene \\
\hline \multirow[t]{12}{*}{ PMR4 } & Citrullus lanatus & & Cucurbita maxima & \\
\hline & ClaPMR4-1 & Cla013561 & CmaPMR4-1 & CmaCh02G000990.1 \\
\hline & ClaPMR4-2 & Cla019505 & CmaPMR4-2 & CmaCh20G002000.1 \\
\hline & ClaPMR4-3 & Cla007811 & CmaPMR4-3 & CmaCh13G006450.1 \\
\hline & ClaPMR4-4 & Cla004782 & CmaPMR4-4 & CmaCh02G006270.1 \\
\hline & ClaPMR4-5 & Cla007809 & CmaPMR4-5 & CmaCh17G009290.1 \\
\hline & Cucurbita pepo & & Cucurbita moschata & \\
\hline & CpePMR4-1 & PU037453 & CmoPMR4-1 & CmoCh02G001000.1 \\
\hline & CpePMR4-2 & PU029642 & CmoPMR4-2 & CmoCh20G002200.1 \\
\hline & CpePMR4-3 & PU034142 & CmoPMR4-3 & CmoCh13G006640.1 \\
\hline & CpePMR4-4 & PU119647 & CmoPMR4-4 & CmoCh02G006310.1 \\
\hline & CpePMR4-5 & PU043411 & CmoPMR4-5 & CmoCh17G009010.1 \\
\hline \multirow[t]{12}{*}{ PMR5 } & Citrullus lanatus & & Cucurbita maxima & \\
\hline & ClaPMR5-1 & Cla008154 & CmaPMR5-1 & CmaCh01G006760.1 \\
\hline & ClaPMR5-2 & Cla013528 & CmaPMR5-2 & CmaCh20G002230.1 \\
\hline & ClaPMR5-3 & Cla009685 & CmaPMR5-3 & CmaCh13G006130.1 \\
\hline & ClaPMR5-4 & Cla005543 & CmaPMR5-4 & CmaCh07G001940.1 \\
\hline & ClaPMR5-5 & Cla005905 & CmaPMR5-5 & CmaCh03G013170.1 \\
\hline & Cucurbita pepo & & Cucurbita moschata & \\
\hline & CpePMR5-1 & PU037441 & CmoPMR5-1 & CmoCh01G007050.1 \\
\hline & CpePMR5-2 & PU059522 & CmoPMR5-2 & CmoCh20G002430.1 \\
\hline & CpePMR5-3 & PU055704 & CmoPMR5-3 & CmoCh13G006370.1 \\
\hline & CpePMR5-4 & PU050925 & CmoPMR5-4 & CmoCh03G013170.1 \\
\hline & CpePMR5-5 & PU041638 & CmoPMR5-5 & CmoCh18G003850.1 \\
\hline \multirow[t]{11}{*}{ PMR6 } & Citrullus lanatus & & Cucurbita maxima & \\
\hline & ClaPMR6-1 & Cla013736 & CmaPMR6-1 & CmaCh04G025490.1 \\
\hline & ClaPMR6-2 & Cla020532 & CmaPMR6-2 & CmaCh15G005000.1 \\
\hline & ClaPMR6-3 & Cla018329 & CmaPMR6-3 & CmaCh18G009110.1 \\
\hline & ClaPMR6-4 & Cla002573 & CmaPMR6-4 & CmaCh04G013920.1 \\
\hline & ClaPMR6-5 & Cla002940 & CmaPMR6-5 & CmaCh07G008920.1 \\
\hline & ClaPMR6-6 & Cla021445 & CmaPMR6-6 & CmaCh03G012030.1 \\
\hline & Cucurbita pepo & & Cucurbita moschata & \\
\hline & CpePMR6-1 & PU055220 & CmoPMR6-1 & CmoCh15G005080.1 \\
\hline & CpePMR6-2 & PU055149 & CmoPMR6-2 & CmoCh04G014650.1 \\
\hline & CpePMR6-3 & PU047808 & CmoPMR6-3 & CmoCh18G009150.1 \\
\hline
\end{tabular}


Citation: Porterfield R, Meru G (2017) Candidate Susceptibility Genes for Powdery and Downy Mildew in Watermelon and Squash. J Phylogenetics Evol Biol 5: 186. doi:10.4172/2329-9002.1000186

Page 9 of 14

\begin{tabular}{|l|l|l|l|l|}
\hline & Homolog & Gene & Homolog & Gene \\
\hline & CpePMR6-4 & PU119980 & CmoPMR6-4 & CmoCh07G009250.1 \\
\hline & CpePMR6-5 & PU049955 & CmoPMR6-5 & CmoCh03G012010.1 \\
\hline & & & CmoPMR6-6 & CmoCh04G026670.1 \\
\hline
\end{tabular}

Table 3: Powdery Mildew Resistance homologs and their designation in the Citrullus lanatus, Cucurbita pepo, Cucurbita maxima and Cucurbita moschata genome databases.

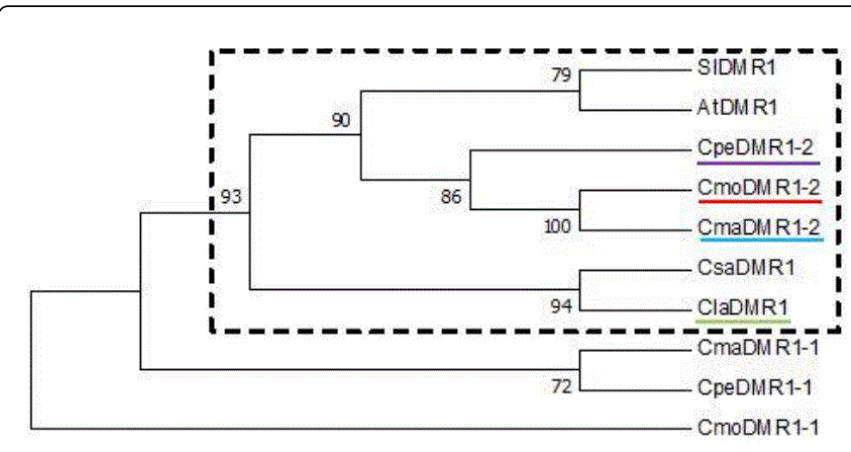

Figure 4A: Downy Mildew Resistance phylogenetic analysis. Phylogenetic tree for DMR1 protein in Arabidopsis (AtDMR1 and AtDMR6) and AtPMR6), tomato (SlDMR1) and cucumber (CsaDMR1, CsaDMR6-1 and CsaDMR6-2) and DMR-like proteins in Citrullus lanatus, Cucurbita pepo, Cucurbita maxima and Cucurbita moschata. Underlined proteins represent those closest in similarity to functionally proven DMR genes (within dotted box).

\section{Chromosomal distribution of MLO, PMR and DMR homologs}

The candidate genes identified in this study were distributed across the $C$. lanatus, $C$. maxima and $C$. moschata genomes (Figure 5A). In watermelon, all the 11 chromosomes had at least one candidate susceptibility gene and clustering of MLO [previously identified by Lovieno et al. [22] and PMR genes was noted on chromosome 3 in an interval $(0.5 \mathrm{Mb}$ to $5.9 \mathrm{Mb})$ known for high nucleotide divergence and enrichment of disease response genes [28] (Figure 5A). Other gene clusters for PMR and DMR homologs were identified on Chromosome 2. In $C$. maxima and $C$. moschata, candidate susceptibility genes were found in all chromosomes except chromosomes 9,10,11 and 12. For the two species, most candidate homologs were located at similar positions on respective chromosomes (Figure 5B-C), thus suggesting high levels of synteny between $C$. maxima and $C$. moschata.

\section{Significance of MLO, PMR and DMR candidate genes}

The current study identified multiple MLO, PMR and DMR candidate gene in $C$. lanatus, $C$. pepo, C. maxima and $C$. moschata which should be investigated to elucidate their role in PM and DM pathogenesis. Approaches such as gene knock out, gene knock down or gene expression analysis will further narrow down the candidate gene list to just a few that can be manipulated through gene-editing methods to create novel PM or DM resistant genotypes.

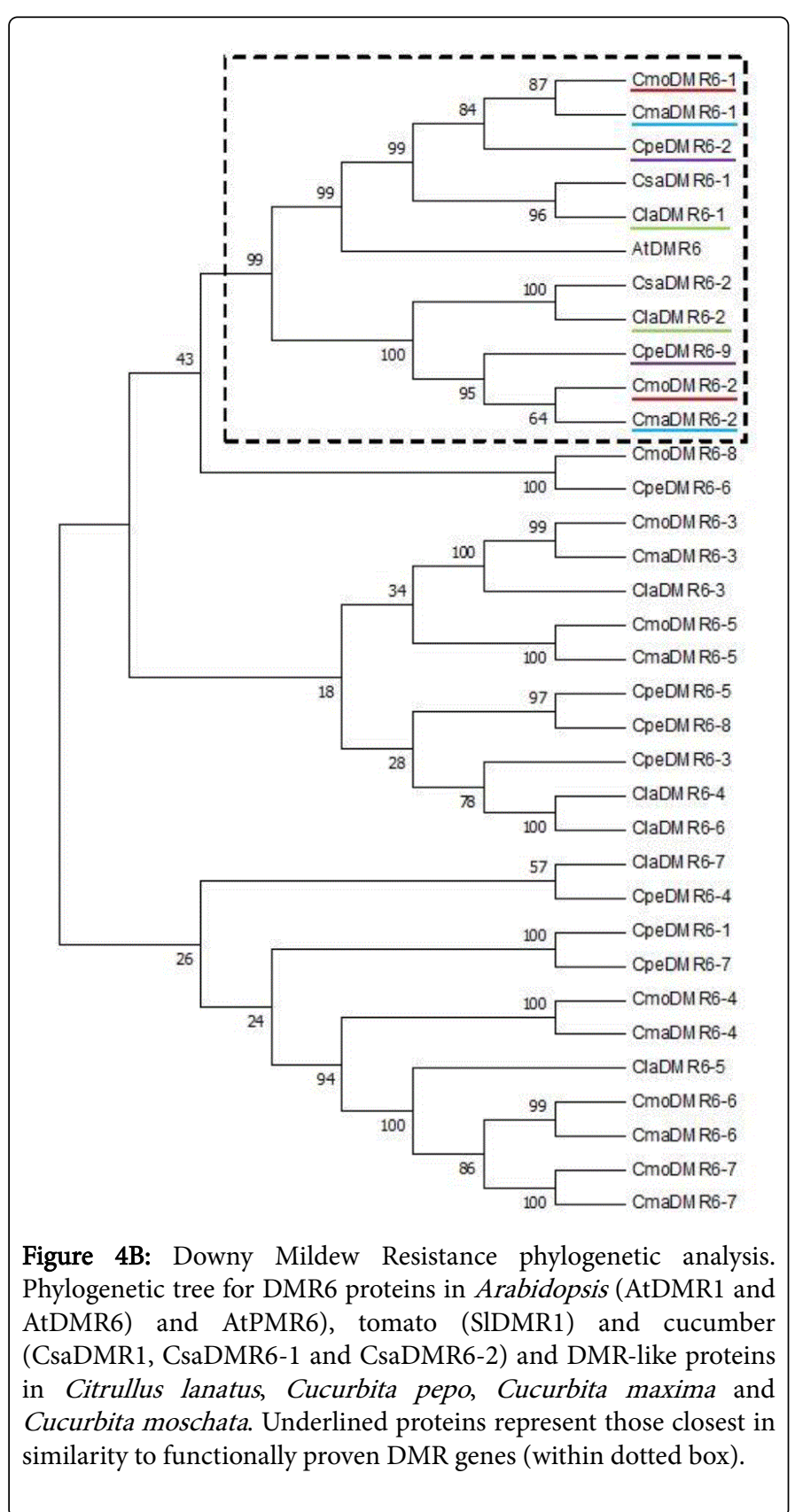


Citation: Porterfield R, Meru G (2017) Candidate Susceptibility Genes for Powdery and Downy Mildew in Watermelon and Squash. J Phylogenetics Evol Biol 5: 186. doi:10.4172/2329-9002.1000186

\begin{tabular}{|c|c|c|c|c|}
\hline & Homolog & Gene & Homolog & Gene \\
\hline \multirow[t]{6}{*}{ DMR1 } & Citrullus lanatus & & Cucurbita maxima & \\
\hline & ClaDMR1 & Cla015737 & CmaDMR1-1 & CmaCh19G008030.1 \\
\hline & Cucurbita pepo & & CmaDMR1-2 & CmaCh06G015720.1 \\
\hline & CpeDMR1-1 & PU030048 & Cucurbita moschata & \\
\hline & CpeDMR1-2 & PU000104 & CmoDMR1-1 & CmoCh19G008250.1 \\
\hline & & & CmoDMR1-2 & CmoCh06G015660.1 \\
\hline \multirow[t]{17}{*}{ DMR6 } & Citrullus lanatus & & Cucurbita maxima & \\
\hline & ClaDMR6-1 & Cla010665 & CmaDMR6-1 & CmaCh16G000150.1 \\
\hline & ClaDMR6-2 & Cla004982 & CmaDMR6-2 & CmaCh15G013330.1 \\
\hline & ClaDMR6-3 & Cla014824 & CmaDMR6-3 & CmaCh08G002970.1 \\
\hline & ClaDMR6-4 & Cla004353 & CmaDMR6-4 & CmaCh16G002270.1 \\
\hline & ClaDMR6-5 & Cla017758 & CmaDMR6-5 & CmaCh05G000430.1 \\
\hline & ClaDMR6-6 & Cla017993 & CmaDMR6-6 & CmaCh14G002480.1 \\
\hline & ClaDMR6-7 & Cla001290 & CmaDMR6-7 & CmaCh06G004160.1 \\
\hline & Cucurbita pepo & & Cucurbita moschata & \\
\hline & CpeDMR6-1 & PU055463 & CmoDMR6-1 & CmoCh16G000180.1 \\
\hline & CpeDMR6-3 & PU057959 & CmoDMR6-2 & CmoCh15G013950.1 \\
\hline & CpeDMR6-4 & PU036758 & CmoDMR6-3 & CmoCh08G002950.1 \\
\hline & CpeDMR6-5 & PU029235 & CmoDMR6-4 & CmoCh16G002480.1 \\
\hline & CpeDMR6-6 & PU041846 & CmoDMR6-5 & CmoCh05G000500.1 \\
\hline & CpeDMR6-7 & PU019772 & CmoDMR6-6 & CmoCh14G002380.1 \\
\hline & CpeDMR6-8 & PU052773 & CmoDMR6-7 & CmoCh06G004140.1 \\
\hline & CpeDMR6-9 & PU135070 & CmoDMR6-8 & CmoCh16G012830.1 \\
\hline
\end{tabular}

Table 4: Downy Mildew Resistance homologs and their designation in the Citrullus lanatus, Cucurbita pepo, Cucurbita maxima and Cucurbita moschata genome databases. 
Citation: Porterfield R, Meru G (2017) Candidate Susceptibility Genes for Powdery and Downy Mildew in Watermelon and Squash. J Phylogenetics Evol Biol 5: 186. doi:10.4172/2329-9002.1000186
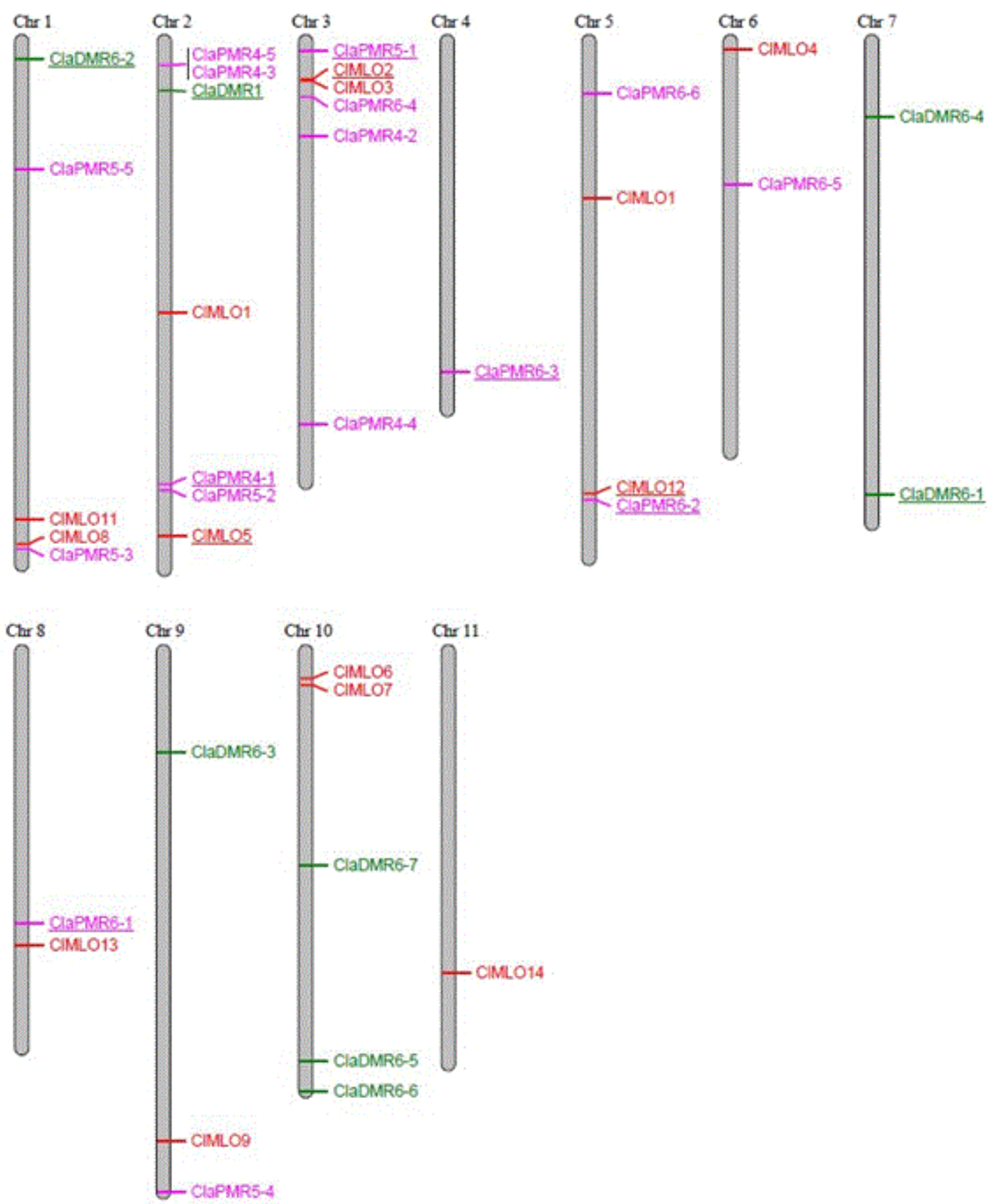

Figure 5A: Physical positions of candidate genes. Distribution of candidate Mildew-locus-O (MLO), Powdery Mildew Resistance (PMR) and Downy Mildew Resistance (DMR) homologs across the genomes for Citrullus lanatus. Citrullus lanatus map includes 14 MLO homologs previously identified by Lovieno et al. [22]. Red, purple and green font represent MLO, PMR and DMR homologs, respectively. Underlined proteins represent those closest in similarity to functionally proven homologs in other species. Chromosomes (Chr) without any candidate genes are not included. 
Citation: Porterfield R, Meru G (2017) Candidate Susceptibility Genes for Powdery and Downy Mildew in Watermelon and Squash. J Phylogenetics Evol Biol 5: 186. doi:10.4172/2329-9002.1000186

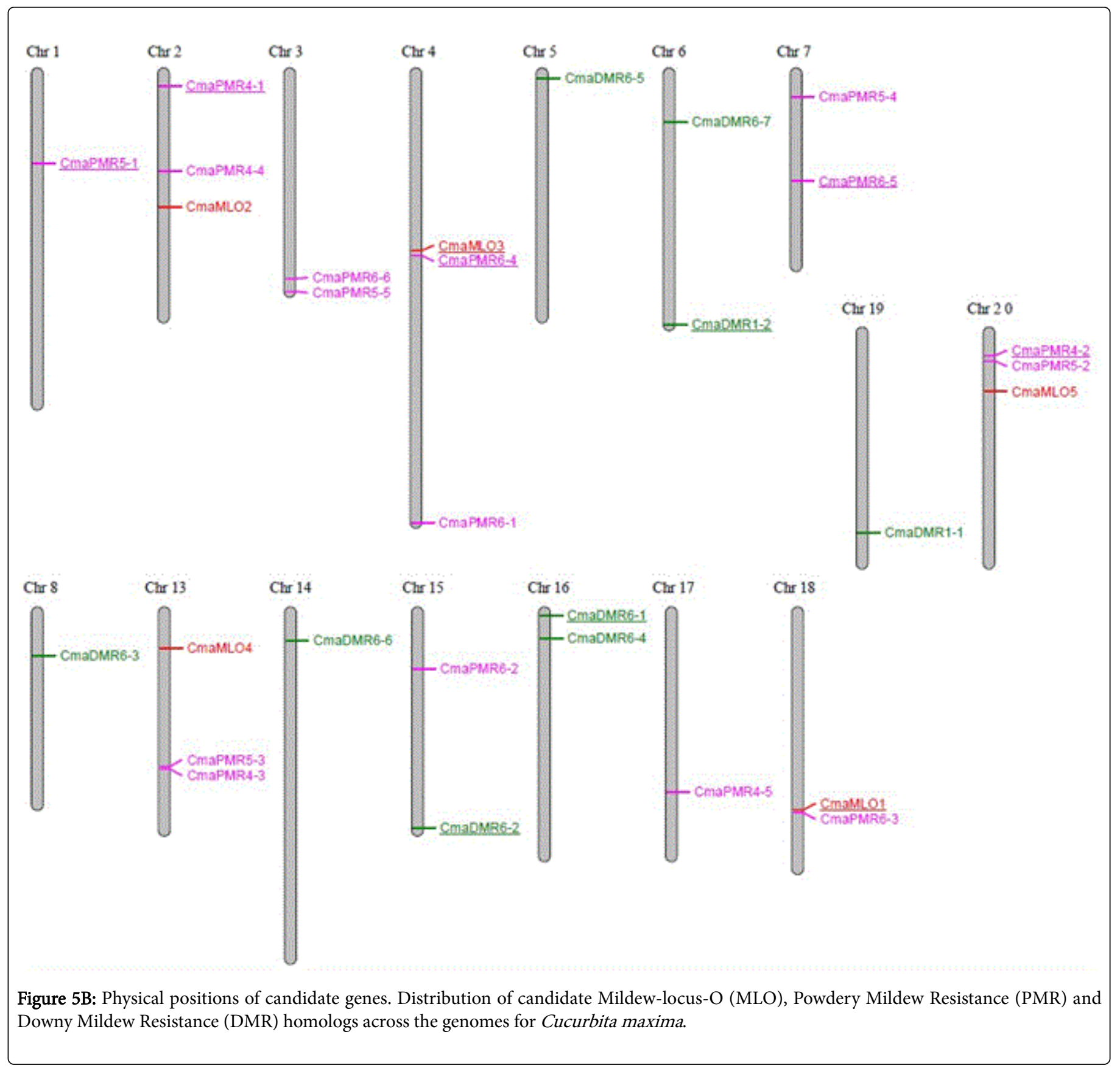


Citation: Porterfield R, Meru G (2017) Candidate Susceptibility Genes for Powdery and Downy Mildew in Watermelon and Squash. J Phylogenetics Evol Biol 5: 186. doi:10.4172/2329-9002.1000186

Page 13 of 14

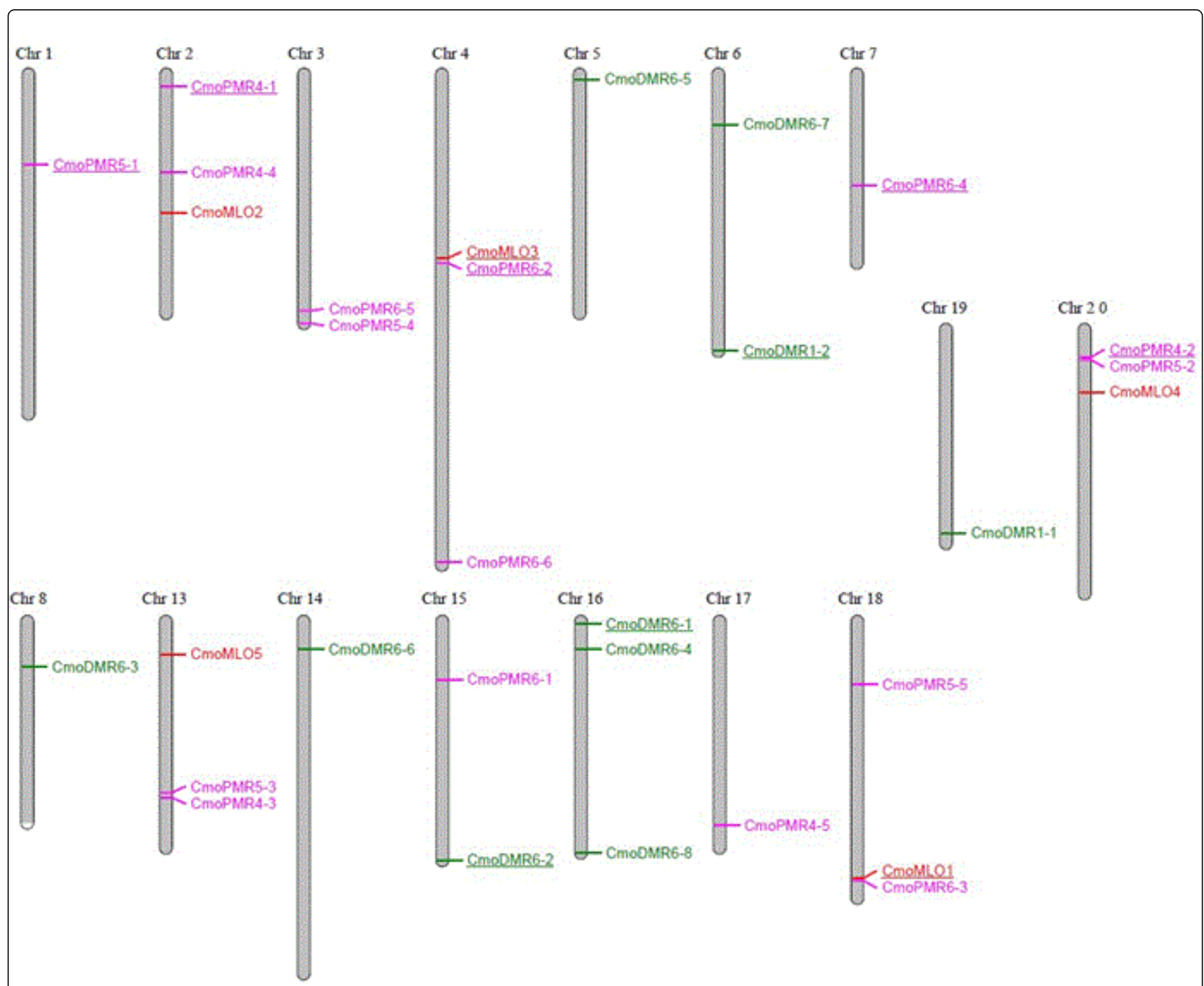

Figure 5C: Physical positions of candidate genes. Distribution of candidate Mildew-locus-O (MLO), Powdery Mildew Resistance (PMR) and Downy Mildew Resistance (DMR) homologs across the genomes for Cucurbita moschata.

\section{References}

1. United States Department of Agriculture (2016) Vegetables 2016 summary In: National Agricultural Statistics Service (eds.), National Agricultural Statistics Service, USA.

2. Braun U, Cook RTA, Inman AJ, Shin HD (2002) The taxonomy of the powdery mildew fungi. American Phytopathological Society 13-55.

3. Zhang S, Mersha Z, Vallad GE, Huang CH (2016) Management of powdery mildew in squash by plant and alga extract biopesticides. Plant Pathol J 32: 528-536.

4. Burns CJ, McIntosh LJ, Mink PJ, Jurek AM, Li AA (2013) Pesticide exposure and neurodevelopmental outcomes: review of the epidemiologic and animal studies. J Toxicol Environ Health B Crit Rev 16: 127-283.

5. Geiger F, Bengtsson J, Berends F, Weisser W, Emmerson M, et al. (2010) Persistent negative effects of pesticides on biodiversity and biological control potential on European farmland. Basic Appl Ecol 11: 97-105.

6. Norgaard KB, Cedergreen N (2010) Pesticide cocktails can interact synergistically on aquatic crustaceans. Environ Sci Pollut Res 17: 957-967.
7. Wang F, Wang C, Liu P, Lei C, Hao W, et al. (2016) Enhanced Rice blast resistance by CRISPR/Cas9-targeted mutagenesis of the ERF transcription factor gene OsERF922. PLoS ONE 11: e0154027.

8. Jia H, Zhang Y, Orbovic V, Xu J, White FF, et al. (2016) Genome editing of the disease susceptibility gene CsLOB1 in citrus confers resistance to citrus canker. Plant Biotechnol J 15: 817-823.

9. Pessina S, Lenzi L, Perazzolli M, Campa M, Costa LD, et al. (2016) Knockdown of MLO genes reduces susceptibility to powdery mildew in grapevine. Hortic Res 16016.

10. Zheng Z, Nonomura T, Appiano M, Pavan S, Matsuda Y, et al. (2013) Loss of function in Mlo orthologs reduces susceptibility of pepper and tomato to powdery mildew disease caused by Leveillula taurica. PLoS ONE 8: e70723.

11. Pessina S, Pavan S, Catalano D, Gallotta A, Visser RG, et al. (2014) Characterization of the MLO gene family in Rosaceae and gene expression analysis in Malus domestica. BMC Genomics 15: 618. 
Citation: Porterfield R, Meru G (2017) Candidate Susceptibility Genes for Powdery and Downy Mildew in Watermelon and Squash. J Phylogenetics Evol Biol 5: 186. doi:10.4172/2329-9002.1000186

Page 14 of 14

12. Schouten HJ, Krauskopf J, Visser R, Bai Y (2014) Identification of candidate genes required for susceptibility to powdery or downy mildew in cucumber. Euphytica 200: 475-486.

13. Kusch S, Pesch L, Panstruga R (2016) Comprehensive phylogenetic analysis sheds light on the diversity and origin of the MLO family of integral membrane proteins. Genome Biol Evol 8: 878-895.

14. Panstruga R (2005) Discovery of novel conserved peptide domains by ortholog comparison within plant multi-protein families. Plant Mol Biol 59: 485-500.

15. Zhang SP, Liu MM, Miao H, Zhang SQ, Yang YH, et al. (2013) Chromosomal mapping and QTL analysis of resistance to downy mildew in Cucumis sativus. Plant Disease 97: 245-251.

16. Nishimura MT, Stein M, Hou BH, Vogel JP, Edwards H, et al. (2003) Loss of a callose synthase results in salicylic acid-dependent disease resistance. Science 301: 969-972.

17. Vogel JP, Raab TK, Schiff C, Somerville SC (2002) PMR6, a pectate lyaselike gene required for powdery mildew susceptibility in Arabidopsis. Plant Cell 14: 2095-2106.

18. Vogel JP, Raab TK, Somerville CR, Somerville SC (2004) Mutations in PMR5 result in powdery mildew resistance and altered cell wall composition. Plant J 40: 968-978.

19. Huibers RP, Loonen AE, Gao D, Van den ackerveken G, Visser RG, et al (2013) Powdery mildew resistance in tomato by impairment of SIPMR4 and SIDMR1. PLoS ONE 8: e67467.

20. van Damme M, Huibers RP, Elberse J, van den Ackerveken G (2008) Arabidopsis DMR6 encodes a putative 2OG-Fe(II) oxygenase that is defense-associated but required for susceptibility to downy mildew. Plant J 54: 785-793.

21. van Damme M, Zeilmaker T, Elberse J, Annemiek Andel, Velde M, et al. (2009) Downy mildew resistance in Arabidopsis by mutation of HOMOSERINE KINASE. Plant Cell 21: 2179-2189.

22. Lovieno P, Andolfo G, Schiavulli A, Catalano D, Ricciardi L, et al. (2015) Structure, evolution and functional inference on the Mildew Locus O (MLO) gene family in three cultivated Cucurbitaceae spp. BMC Genomics 16: 1112 .

23. Voorrips RE (2002) MapChart: Software for the graphical presentation of linkage maps and QTLs. J Hered 93: 77-78.

24. Tamura K, Peterson D, Peterson N, Stecher G, Nei M, et al. (2011) MEGA5: molecular evolutionary genetics analysis using maximum likelihood, evolutionary distance, and maximum parsimony methods. Mol Biol Evol 28: 2731-2739.

25. Devoto A, Hartmann HA, Piffanelli P, Elliott C, Simmons C, et al. (2003) Molecular phylogeny and evolution of the plant-specific seventransmembrane MLO family. J Mol Evol 56: 77-88.

26. Devoto A, Piffanelli P, Nilsson I, Wallin E, Panstruga R, et al. (1999) Topology, subcellular localization, and sequence diversity of the Mlo family in plants. J Biol Chem 274: 34993-35004.

27. Sayle R (1994) RasMol v 2.6 manual. http://life.nthu.edu.tw/ ${ }^{*} \mathrm{fmhsu} /$ rasframe/COLORS.HTM\#aminoc.

28. Guo S, Zhang J, Sun H, Salse J, Lucas WJ, et al. (2013) The draft genome of watermelon (Citrullus lanatus) and resequencing of 20 diverse accessions. Nature Genet 45: 51-58. 